\title{
Staging Laparoscopy in Patients with Intrahepatic Cholangiocarcinoma: Is It Still Useful?
}

\author{
Lotte C. Franken $^{a}$ Robert Jan S. Coelen ${ }^{a} \quad$ Eva Roos $^{a, b} \quad$ Joanne Verheij ${ }^{b}$ \\ Saffire S. Phoac Marc G. Besselink ${ }^{a}$ Olivier R.C. Busch ${ }^{a}$ Thomas M. van Gulik ${ }^{a}$ \\ aDepartment of Surgery, Cancer Center Amsterdam, Amsterdam UMC, University of Amsterdam, Amsterdam, The \\ Netherlands; ${ }^{b}$ Department of Pathology, Cancer Center Amsterdam, Amsterdam UMC, University of Amsterdam, \\ Amsterdam, The Netherlands; ${ }^{\circ}$ Department of Radiology and Nuclear Medicine, Amsterdam UMC, University of \\ Amsterdam, Amsterdam, The Netherlands
}

\section{Keywords}

Intrahepatic cholangiocarcinoma · Yield · Sensitivity · Resectability · Staging laparoscopy

\begin{abstract}
Background: The role of staging laparoscopy in patients with intrahepatic cholangiocarcinoma remains unclear. Despite extensive preoperative imaging, approximately $25 \%$ of patients are deemed unresectable at laparotomy due to metastasized disease. The aim of this study was to evaluate the frequency of unresectable disease found at staging laparoscopy and to identify predictors for detecting metastasized intrahepatic cholangiocarcinoma. Methods: We retrospectively collected records of all patients with intrahepatic cholangiocarcinoma, presenting at our institution from 2008 to 2017. Staging laparoscopy was performed on the suspicion of distant metastases and on indication in larger tumors. The yield and sensitivity of staging laparoscopy was calculated. Reasons for unresectability at staging laparoscopy or laparotomy were recorded. Results: Among a total of 80 patients with potentially resectable intrahepatic cholangiocarcinoma, 35 patients underwent staging laparoscopy on the suspicion of distant metastases. Unresectable disease was found at staging laparoscopy in 15 patients. Reasons for unresectability were liver metastasis $(n=6)$, peritoneal metas-
\end{abstract}

karger@karger.com www.karger.com/vis

Karger BOPEN ACCESS
(C) 2020 The Author(s)

Published by S. Karger AG, Basel

This article is licensed under the Creative Commons AttributionNonCommercial-NoDerivatives 4.0 International License (CC BYNC-ND) (http://www.karger.com/Services/OpenAccessLicense) Usage and distribution for commercial purposes as well as any disUsage and distribution for commercial purposes as well as any tasis $(n=4)$, severe cirrhosis $(n=2)$, locally advanced tumor with satellite lesions $(n=1)$, and distant lymph node metastasis $(n=2)$. Considering optimal preoperative imaging, the true yield of staging laparoscopy was $20 \%$ (7/35). Two patients did not undergo laparotomy due to progression after staging laparoscopy. Of the remaining 18 patients who underwent laparotomy, 6 patients (30\%) had unresectable disease, mostly because of distant metastasis $(n=4)$. Conclusions: The role of staging laparoscopy to detect unresectable intrahepatic cholangiocarcinoma is highly dependent on the quality of preoperative imaging. Currently, no accurate selection criteria on imaging exist to select patients with intrahepatic cholangiocarcinoma who potentially benefit from staging laparoscopy.

(C) 2020 The Author(s) Published by S. Karger AG, Basel

\section{Introduction}

Intrahepatic cholangiocarcinoma (IHC) is a rare tumor originating in the peripheral bile ducts within the liver parenchyma, usually only causing symptoms at a late stage. After hepatocellular carcinoma, cholangiocarcinoma is the second most common primary hepatic malignancy. The incidence of intrahepatic malignancies varies widely between countries and accounts for $5-10 \%$ of all cholangio- 
Table 1. Patient characteristics in staging laparoscopy and explorative laparotomy

\begin{tabular}{llll}
\hline & $\begin{array}{l}\text { Staging laparoscopy } \\
(n=35)\end{array}$ & $\begin{array}{l}\text { Explorative laparotomy } \\
\text { without staging laparoscopy } \\
(n=45)\end{array}$ & $p$ value \\
\hline Female gender & $16(46)$ & $22(49)$ & 0.778 \\
Age, years & $64 \pm 9$ & $65 \pm 10$ & 0.608 \\
Tumor size, cm & $5.5(3.8-9.0)(n=31)$ & $3.5(2.2-7.1)(n=42)$ & 0.026 \\
Imaging & $12(34)$ & $15(33)$ & 0.655 \\
$\quad$ CT only & 0 & $1(2)$ & 0.929 \\
$\quad$ MRI only & $23(66)$ & $29(64)$ & 0.375 \\
$\quad$ CT and MRI & $10(29)$ & $13(29)$ & 0.906 \\
$\quad$ PET & $21 / 35(60)$ & $14 / 44(31)$ & 0.975 \\
Vascular involvement on imaging & & 0.012 \\
\hline
\end{tabular}

Data are presented as $n(\%)$, mean $\pm \mathrm{SD}$, or median (IQR), as appropriate. CT, computer tomography; MRI, magnetic resonance imaging; PET, positron emission tomography.

carcinoma in Western countries $[1,2]$. The only cure for IHC is radical surgical resection. Despite improvements in preoperative imaging, a subset of patients presents with unresectable disease at explorative laparotomy. Previous reports dealing with resectable IHC have shown that resection is feasible in only $30-40 \%$ of the patients undergoing explorative laparotomy. Reasons for unresectability in these patients were metastases (liver, nodal, or peritoneal) or locally advanced disease $[3,4]$. Staging laparoscopy (SL) potentially detects unresectability and may avoid unnecessary laparotomy. By avoiding a futile laparotomy, patients benefit from earlier referral for systemic treatment, decreased hospital stay, and less postoperative pain. However, the usefulness of SL in patients with potentially resectable IHC is debated and no reliable criteria exist to select patients for SL. The reported yield varies from 11 to $36 \%$, with the largest series including 44 patients with IHC [5-7]. In the present study, we aimed to evaluate the frequency of unresectable disease found at SL in patients with IHC considered resectable on preoperative imaging.

\section{Materials and Methods}

A retrospective observational cohort study was conducted. We collected records of patients presenting with potentially resectable IHC between 2008 and 2017 at our tertiary center. Patients' demographic data, preoperative imaging, pathology reports, operation reports, and complications of treatment were recorded. For imaging, computer tomography (CT) scans, magnetic resonance imaging (MRI) and/or positron emission tomography (PET) scans were used. Standard CT liver protocol included a dynamic contrast-enhanced CT with arterial and venous phase. Standard MRI liver protocol is at $1.5 \mathrm{~T}$, dynamic 4-phase contrast-enhanced MRI with gadolinium (T2-weighted and diffusion-weighted imaging [b50, b400, b800]). All patients were discussed by a dedicated hepatopancreato-biliary multidisciplinary team, consisting of experienced oncologists, gastroenterologists, radiologists, and specialized hepato-pancreato-biliary surgeons. Based on all relevant im- aging, resectability was assessed and patients were selected for SL or laparotomy. Reasons to perform SL were suspicion of metastatic disease that could not be confirmed by biopsy (lymphadenopathy, liver and/or satellite lesions) and larger tumors $(>5 \mathrm{~cm})$ on imaging. For patients undergoing SL and/or laparotomy, reasons for selecting patients were recorded, as well as reasons for unresectability at SL or laparotomy and complications occurring after SL. At SL, thorough inspection of the abdominal cavity, liver, and diaphragm took place and biopsies of any suspicious lesions were performed. The frequency of unresectable disease at SL was registered. The "true" yield of SL was defined as the proportion of laparotomies avoided in patients with optimal imaging, which was calculated by dividing the number of avoided laparotomies by the total number of performed laparoscopies. Sensitivity was defined as the probability of detecting unresectable disease and was calculated by dividing the number of unresectable patients at SL (true positives) by the total number of patients with unresectable disease (true positives plus false negatives). Statistical analyses were performed using IBM SPSS Statistics version 24.0. A sensitivity analysis was performed, excluding patients in whom preoperative imaging only consisted of CT (and no MRI was performed).

\section{Results}

\section{Patient Demographics and Imaging}

A total of 193 patients with suspected IHC were identified between November 2008 and December 2017, of whom 80 patients presented with potentially resectable disease. Patient characteristics are listed in Table 1. Imaging consisted of multi-phase liver CT scan plus MRI in 52 patients, CT scan only in 27 patients, and MRI only in 1 patient. PET scan was performed in 23 patients. SL was performed in 35 out of 80 patients (44\%) and 45 out of 80 patients $(56 \%)$ underwent laparotomy without SL. The proportion of patients undergoing CT as well as MRI was $22 / 35(65 \%)$ in patients with SL and 29/45 (65\%) in patients with laparotomy without SL. A flowchart of all patients is displayed in Figure 1. 
Fig. 1. Flowchart of all patients with potentially resectable intrahepatic cholangiocarcinoma.

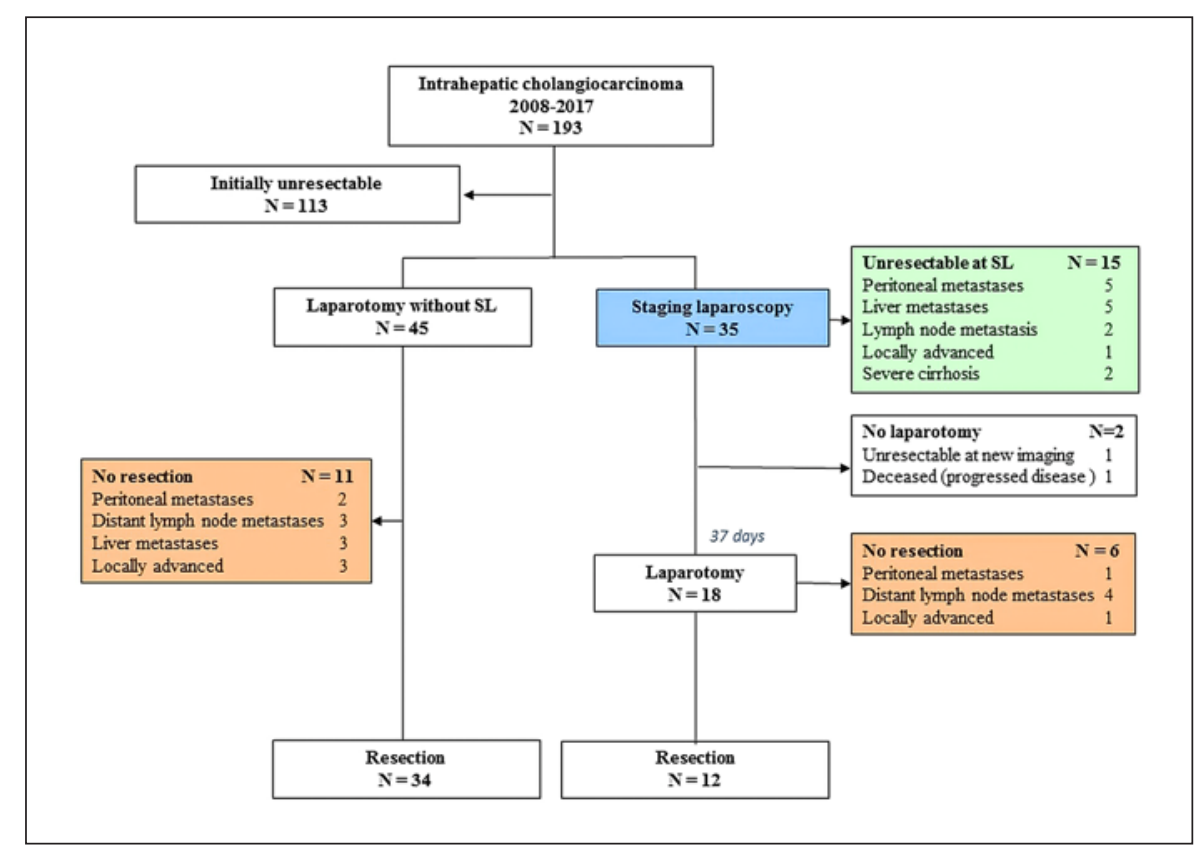

In the majority of the 35 patients undergoing SL, the procedure was performed on the suspicion of metastatic disease $(n=21)$. In these patients, lymph node or liver metastasis could not be confirmed by biopsy. Other reasons for performing SL were tumor size $>5 \mathrm{~cm}(n=5)$, pathologic confirmation of diagnosis $(n=7)$, and assessment of the quality of the future remnant liver in 2 patients with cirrhosis (aided by intraoperative biopsies).

\section{Surgical Findings}

Of all 35 patients undergoing SL, unresectable disease was detected in 15 patients at SL (see Fig. 1). Laparoscopic ultrasound was performed in 2 patients. Intra-operative biopsies were performed in 28 out of 35 patients $(80 \%)$, resulting in confirmation of malignancy in $12 \mathrm{pa}-$ tients. Two intraoperative biopsies of liver parenchyma confirmed severe cirrhosis. Reasons for unresectability at SL were liver metastasis $(n=6)$, peritoneal metastasis $(n=4)$, severe cirrhosis $(n=2)$, locally advanced tumor with satellite lesions $(n=1)$, and distant lymph node metastasis (station 8$)(n=2)$. Out of 15 patients, preoperative imaging included an MRI in 9 patients (60\%). The true yield of SL, considering optimal use of imaging modalities, was $20 \%$ (7 out of 35 ).

In only 1 patient (3\%), SL was complicated by a serosal tear of the small bowel, which was managed by simple suturing. Two patients who were considered resectable at SL did not undergo explorative laparotomy due to progression of disease after SL. One patient had undergone arterial embolization of the tumor and portal vein embolization after SL. A CT scan performed 46 days after SL showed new lesions in the future remnant liver, highly suspicious of liver metastases. The other patient devel- oped jaundice shortly after SL. This was treated by percutaneous biliary drainage, complicated by cholangiosepsis and multiple organ failure leading to his demise 19 days after SL. The median interval between SL and laparotomy was 37 days. Of the remaining 18 patients who underwent explorative laparotomy after SL, 6 patients were found to have unresectable IHC. Reasons for unresectability in this group were peritoneal metastases $(n=1)$, lymph node metastases $(n=4)$, and locally advanced tumor $(n=1)$. The overall sensitivity of SL for unresectability was $71 \%$ (15 out of 21). Of the 45 patients who underwent explorative laparotomy without SL, 34 patients had resectable disease whereas 11 patients were diagnosed with unresectable tumors. Reasons for unresectability in this group were peritoneal metastases $(n=2)$, distant lymph node metastases $(n=3)$, liver metastases $(n=3)$, and locally advanced disease $(n=3)$. Overall, 63 laparotomies were performed, which led to 46 resections (73\%).

\section{Discussion}

This is the largest clinical series of SL in IHC to date showing that in patients with presumed resectable IHC, the role of SL has not been replaced by imaging completely. Considering optimal preoperative imaging, the "true" yield of SL for detecting unresectable IHC was 20\% (7/35).

The yield of SL, of course, is highly dependent on optimal use of imaging. Improved imaging techniques may have led to decreased yield in hepatobiliary malignancies, for example as has been observed in pancreatic tumors. This decrease may continue as imaging techniques develop and evolve, as seen by the introduction of hepato- 
Table 2. Characteristics of studies reporting on yield and accuracy of staging laparoscopy in intrahepatic cholangiocarcinoma

\begin{tabular}{|c|c|c|c|c|c|c|}
\hline Author & Journal & Year & Patients & $\begin{array}{l}\text { Patients, } \\
n\end{array}$ & $\begin{array}{l}\text { Yield, } \\
n(\%)\end{array}$ & $\begin{array}{l}\text { Accuracy/ } \\
\text { sensitivity, } \\
n(\%)\end{array}$ \\
\hline Weber et al. [7] & J Am Coll Surg & 2001 & IHC & 22 & $27(6 / 22)$ & $55(6 / 11)$ \\
\hline Goere et al. [5] & Surg Endosc & 2005 & $\begin{array}{l}\text { Biliary cancer } \\
\text { IHC }\end{array}$ & $\begin{array}{l}39 \\
11\end{array}$ & $\begin{array}{l}38(14 / 39) \\
36(4 / 11)\end{array}$ & $\begin{array}{l}61(14 / 23) \\
67(4 / 6)\end{array}$ \\
\hline Russolillo et al. [6] & Surg Endosc & 2016 & $\begin{array}{l}\text { Proximal biliary cancer } \\
\text { IHC }\end{array}$ & $\begin{array}{r}100 \\
44\end{array}$ & $\begin{array}{l}18(18 / 100) \\
11.4(5 / 44)\end{array}$ & $\begin{array}{r}60(18 / 30) \\
62.5(5 / 8)\end{array}$ \\
\hline
\end{tabular}

IHC, intrahepatic cholangiocarcinoma.

biliary contrast-enhanced MRI proving to have a high sensitivity in detecting hepatic metastases [8]. In our series, not all patients underwent preoperative staging MRI, and therefore preoperative imaging may not have been optimal. This is why we have defined the "true" yield of SL, considering optimal imaging, rather than the overall yield of detecting unresectable disease (15 out of 35). Out of these 15 unresectable patients, 7 patients were considered "true" yield of SL, of whom 6 had undergone an MRI. To date, CT and MRI are considered to have comparable sensitivity in the detection of peritoneal metastases [9]. Therefore, in one patient with suspected peritoneal metastases on CT that had to be confirmed by SL, this was considered "true" yield of SL. By not including other patients without preoperative MRI in the true yield of SL $(20 \%)$, it is possible that the current yield is actually an underestimation of the yield if optimal imaging was applied in all patients. For example, if small intrahepatic lesions $(<3 \mathrm{~mm})$ were detected by the MRI, it is possible that these could only be confirmed by SL.

Unfortunately, no reliable criteria are available to select patients with IHC who potentially benefit from SL. In our series, SL was performed on the suspicion of metastatic disease (lymphadenopathy or/and liver lesions) and on indication in larger tumors $(>5 \mathrm{~cm})$. However, there were patients with tumors $>5 \mathrm{~cm}$ who underwent laparotomy without SL. Of the 45 patients who underwent upfront laparotomy, 11 patients were still deemed unresectable on the basis of intraoperative findings. These findings highlight that the present criteria for selecting patients for SL were not sufficient to select all patients who could potentially benefit from SL. Also, the median interval of 37 days between SL and laparotomy could have affected the sensitivity.

In the literature, the reported accuracy of SL varies widely among different types of biliary cancers [5]. A recent meta-analysis on the diagnostic impact of SL in patients with perihilar cholangiocarcinoma ( $\mathrm{PHC}$ ) and gallbladder cancer showed a pooled yield of 32.4 and $27.6 \%$, respectively [10]. A meta-analysis conducted by our group showed a pooled yield of SL of $24.4 \%$ in patients with PHC [11]. It has also been shown that the sensitivity of SL differs widely among the various diagnostic predictors, depending on the selection of patients. Bird et al. [12] reported in 114 patients with PHC a 66\% overall sensitivity to detect unresectable disease, with sensitivities to detect peritoneal metastasis and intrahepatic metastasis of 71 and $43 \%$, respectively. Our group has previously reported an overall yield of $15 \%$ in unselected patients with PHC. In these patients, locally advanced disease and lymph node metastases were most difficult to identify at SL [13].

Reports on the role of SL in patients with IHC are scarce. Results of three studies reporting on the yield are shown in Table 2. A small study including 22 patients with IHC reported a $27 \%$ yield and sensitivity of $55 \%$ [7]. A $36 \%$ yield in 11 IHC patients was found in another small series [5].

Russolillo et al. [6] investigated the additional value of laparoscopic ultrasound during SL in patients with proximal biliary cancers, including 44 IHC patients. They reported a $11.4 \%$ yield without the use of ultrasound that increased to $19 \%$ when intraoperative ultrasound was used. A previous study in our institution showed limited additional value of laparoscopic ultrasound in patients with malignant proximal bile duct obstruction [14]. Therefore, laparoscopic ultrasound was only selectively performed in 2 patients in this study.

The retrospective design of this study, a major limitation, made it difficult to retrieve case-by-case considerations at our multidisciplinary team meeting in detail. Selection bias associated with our single-center retrospective study is therefore another limitation of this study. Therefore, our reported "true" yield of $20 \%$ might not be applicable to all settings and reproducibility majorly depends on the selection of patients. Another limitation is the limited number of patients included. Due to the small number of patients it was not possible to identify predic- 
tors for unresectable disease at SL. If such predictors are not found one could also argue that exploration for IHC should start with standard SL in the same procedure. A downside of this practice would be the time needed for a definitive pathological result as frozen sections of peritoneal, lymph node metastases and peritoneal lesions are often inconclusive.

Although evolving imaging techniques may lead to a decreased yield of SL over time, to date imaging has not completely replaced SL in detecting unresectable IHC. Larger studies are necessary to identify predictors of unresectable disease on imaging.

\section{Statement of Ethics}

The need for ethical approval was waived by the Medical Ethics Review Committee of the Amsterdam UMC.

\section{Disclosure Statement}

The authors have no conflicts of interest to declare.

\section{Funding Sources}

The authors have no funding sources to declare.

\section{Author Contributions}

L.C. Franken and R.J.S. Coelen: analysis and interpretation of data, and drafting the work. E. Roos: acquisition of data and revising the work for important content. J. Verheij, M.G. Besselink, T.M. van Gulik, and O.R.C. Busch: design of the study, interpretation of the data, and revising the work for important intellectual content. S.S. Phoa: revising the work for important content and interpretation of the data.

All the above authors approved the final version to be published and agreed to be accountable for all aspects of the work in ensuring that questions related to the accuracy or integrity of any part of the work are appropriately investigated and resolved.

\section{References}

1 Khan SA, Thomas HC, Davidson BR, TaylorRobinson SD. Cholangiocarcinoma. Lancet. 2005 Oct;366(9493):1303-14.

2 Nakeeb A, Pitt HA, Sohn TA, Coleman J, Abrams RA, Piantadosi S, et al. Cholangiocarcinoma. A spectrum of intrahepatic, perihilar, and distal tumors. Ann Surg. 1996 Oct;224(4): 463-73; discussion 473-5.

3 Endo I, Gonen M, Yopp AC, Dalal KM, Zhou $\mathrm{Q}$, Klimstra D, et al. Intrahepatic cholangiocarcinoma: rising frequency, improved survival, and determinants of outcome after resection. Ann Surg. 2008 Jul;248(1):84-96.

4 Tan JC, Coburn NG, Baxter NN, Kiss A, Law $\mathrm{CH}$. Surgical management of intrahepatic cholangiocarcinoma-a population-based study. Ann Surg Oncol. 2008 Feb;15(2):600-8.

5 Goere D, Wagholikar GD, Pessaux P, Carrère $\mathrm{N}$, Sibert A, Vilgrain V, et al. Utility of staging laparoscopy in subsets of biliary cancers : laparoscopy is a powerful diagnostic tool in patients with intrahepatic and gallbladder carcinoma. Surg Endosc. 2006 May;20(5):721-5.
6 Russolillo N, D'Eletto M, Langella S, Perotti S, Lo Tesoriere R, Forchino F, et al. Role of laparoscopic ultrasound during diagnostic laparoscopy for proximal biliary cancers: a single series of 100 patients. Surg Endosc. 2016 Mar; 30(3):1212-8.

7 Weber SM, Jarnagin WR, Klimstra D, DeMatteo RP, Fong Y, Blumgart LH. Intrahepatic cholangiocarcinoma: resectability, recurrence pattern, and outcomes. J Am Coll Surg. 2001 Oct;193(4):384-91.

8 Lafaro KJ, Roumanis P, Demirjian AN, Lall C, Imagawa DK. Gd-EOB-DTPA-enhanced MRI for detection of liver metastases from colorectal cancer: a surgeon's perspective! Int J Hepatol. 2013;2013:572307.

9 Patel CM, Sahdev A, Reznek RH. CT, MRI and PET imaging in peritoneal malignancy. Cancer Imaging. 2011 Aug;11(1):123-39.

10 Tian Y, Liu L, Yeolkar NV, Shen F, Li J, He Z. Diagnostic role of staging laparoscopy in a subset of biliary cancers: a meta-analysis. ANZ J Surg. 2017 Jan;87(1-2):22-7.
11 Coelen RJ, Ruys AT, Besselink MG, Busch OR, van Gulik TM. Diagnostic accuracy of staging laparoscopy for detecting metastasized or locally advanced perihilar cholangiocarcinoma: a systematic review and metaanalysis. Surg Endosc. 2016 Oct;30(10):416373 .

12 Bird N, Elmasry M, Jones R, Elniel M, Kelly M, Palmer D, et al. Role of staging laparoscopy in the stratification of patients with perihilar cholangiocarcinoma. Br J Surg. 2017 Mar;104(4):418-25.

13 Coelen RJ, Ruys AT, Wiggers JK, Nio CY, Verheij J, Gouma DJ, et al. Development of a Risk Score to Predict Detection of Metastasized or Locally Advanced Perihilar Cholangiocarcinoma at Staging Laparoscopy. Ann Surg Oncol. 2016 Dec;23(S5 Suppl 5):904-10.

14 Tilleman EH, de Castro SM, Busch OR, Bemelman WA, van Gulik TM, Obertop H, et al. Diagnostic laparoscopy and laparoscopic ultrasound for staging of patients with malignant proximal bile duct obstruction. J Gastrointest Surg. 2002 May-Jun;6(3):426-30; discussion $430-1$. 